\section{Starvation associated refeeding syndrome in missing cats: 10 cases}

\section{Emily Whitby ${ }^{1}$, Neus Elias ${ }^{2}$, Georgina Hall ${ }^{3}$, Simon Cook ${ }^{1}$}

1 Department of Clinical Sciences and Services, Queen Mother Hospital for Animals, Royal Veterinary College, North Mymms, Hertfordshire, United Kingdom

2 Vets Now Glasgow Hospital, Glasgow, United Kingdom

3 Royal (Dick) School of Veterinary Studies, University of Edinburgh, Edinburgh, United Kingdom

\section{OBJECTIVES}

The aims of this report are to describe the clinicopathological findings associated with refeeding syndrome, and to gain a better understanding of disease presentation, prognostication and prevention in cats.

\section{METHODS}

Computerised records from four referral hospitals were searched between May 2013 and June 2019 inclusive, and ten cases were identified that were eligible for inclusion.

\section{RESULTS}

All cats had been missing for a period of three weeks or more prior to hospitalisation. Cats were missing for a median of 6 weeks $(3-104)$ prior to hospitalisation. Cats were hospitalised for a mean of $15( \pm 5)$ days. $90 \%$ of cats became hypophosphataemic during hospitalisation, with a mean lowest serum phosphorus concentration of 0.89 $( \pm 0.27) \mathrm{mmol} / \mathrm{L}$. All cats became hypokalaemic, with a mean lowest serum potassium concentration of 2.62 $( \pm 0.42) \mathrm{mmol} / \mathrm{L}$. All cats received phosphorus, potassium, magnesium and thiamine supplementation. $60 \%$ of cats were documented as hyperglycaemic, and $60 \%$ were documented as hypoglycaemic, with $20 \%$ exhibiting both. All cats developed anaemia, with a mean lowest PCV of $14.6 \%( \pm 3.81 \%)$. Subsequently, $60 \%$ of cats received a blood transfusion. $3 / 10$ cats had lost over $50 \%$ of their body weight, two of which survived to discharge. $80 \%$ of cats survived to discharge.

\section{STATEMENT (CONCLUSIONS)}

Cats presenting after prolonged starvation and extensive weight loss with refeeding associated complications appear to have a good prognosis but require extensive hospitalisation and intensive care.

\section{Red blood cell trauma during canine blood transfusion; a comparison of two infusion methods}

\section{Melissa White, Simon Tappin}

Dick White Referrals, Six Mile Bottom, United Kingdom

\section{OBJECTIVES}

To determine whether the use of a peristaltic infusion pump (Braun Infusomat Space $®$ ) causes additional mechanical trauma to canine red bloods cells in comparison to using a manually controlled gravity giving set.

\section{METHODS}

A canine blood transfusion was simulated by simultaneously running canine packed red blood cells through a gravity giving set and a peristaltic transfusion pump at the same rate. A sample of the blood was taken pre and post transfusion and run through the ADVIA 120 haematology analyser ${ }^{\circledR}$. The free haemoglobin (FHGB) levels were then calculated and these used to compare the level of haemolysis occurring in each method.

\section{RESULTS}

Ten blood samples were analysed using both methods. The results were divided into three groups; pretransfusion FHGB, gravity giving set FHGB and peristaltic pump FHGB. These groups were then compared using a Friedman test followed by Dunn's post-hoc multiple comparisons. There was a statistically significant increase in FHGB from the pre transfusion samples compared to post both post gravity giving set method $(p=0.01)$ and post transfusion pump method $(P=0.03)$. There was no statistically significant difference in the $\mathrm{FHGB}$ when comparing the two transfusion methods $(P=>0.99)$.

\section{STATEMENT (CONCLUSIONS)}

This study suggests that there is no difference in the level of haemolysis caused by the two transfusion methods, thus suggesting that this peristaltic infusion pump could be used as an alternative method for delivering canine blood transfusions. 